\title{
DESIGN AND IMPLEMENTATION OF A RECONFIGURABLE FIR FILTER
}

\author{
Kuan-Hung Chen and Tzi-Dar Chiueh \\ Graduate Institute of Electronics Engineering and Department of Electrical Engineering, \\ National Taiwan University, Taipei, Taiwan 10617
}

\begin{abstract}
Finite impulse response (FIR) filters are very important blocks in digital communication systems. Many efforts have been made to improve the filter performance, e.g., less hardware and higher speed. In addition, software radio has recently gained much attention due to the need for integrated and reconfigurable communication systems. To this end, reconfigurability has become an important issue for the future filter design. In this paper, we present a digitreconfigurable FIR filter architecture with the finest granularity. The proposed architecture is implemented in a singlepoly quadruple-metal $0.35-\mu \mathrm{m}$ CMOS technology. Measurement results show that the fabricated chip consumes $16.5 \mathrm{~mW}$ of power when operating at $86 \mathrm{MHz}$ under 2.5 $\mathrm{V}$.
\end{abstract}

\section{INTRODUCTION}

Finite impulse response (FIR) filters are important blocks in digital communication systems. These filters can be used to perform a wide variety of tasks such as spectral shaping, matched filtering, noise rejection, channel equalization, etc. As such, various architectures and implementation methods have been proposed to improve the performance of these filters in speed and complexity.

However, with explosive proliferation in communication standards, traditional hardwired devices may not be suitable for future communication needs. Software radio $[1,2]$ has gained much attention from the researchers worldwide due to a unanimous demand for integrated reconfigurable communication systems. Therefore, in the future, not only the speed and the complexity of filters, but also their programmability and reconfigurability should be considered.

It is well known that the canonical signed digit (CSD) representation can be used to reduce the complexity of FIR digital filter implementation $[3,4,5]$. Encoding the filter coefficients using the CSD representation reduces the number of partial products as well as silicon area and power consumption. Hence, it is a useful technique for implementation of FIR filters with fixed coefficients.

This work was supported in part by MediaTek Inc.
While applying the CSD representation to the implementation of programmable FIR filters, it is straightforward to require all filter taps to have the same number of programmable CSDs. However, for most filters, many taps do not require the highest coefficient precision. Valuable hardware resources will be wasted if all taps are implemented with the highest precision. To minimize the computational resources, there have been some works $[6,7]$ implementing programmable FIR filters by restricting the number of nonzero CSDs in each tap. However, the restriction lowers the coefficient precision and may degrade the performance of the filter. Since there are usually some taps that require fewer CSDs, computational resources are still wasted. Another hardware-efficient implementation of programmable FIR filters with CSD coefficients has been presented in [8]. A 32-tap linear-phase filter, with 2 nonzero CSDs in each tap, is implemented. Additional nonzero CSDs can be allocated to specific filter taps, making it a reconfigurable FIR filter architecture. Nevertheless, some computational resources are unused and the critical path can be quite long in some cases.

Due to the wide range of the filter coefficient precision for different applications, it is not easy to achieve reconfigurability without sacrificing simplicity. As an example, a matched filter that is very important for CDMA-based systems requires only 1-bit coefficient precision but a pulseshaping filter may require as high a precision as 16 bits. Since the tap complexity in these two cases can be quite different, it is not efficient to construct a tap-based reconfigurable FIR filter.

In this paper, we adopt a fine granularity for filter implementation and develop a digit-reconfigurable FIR filter architecture with extreme flexibility. With this architecture, both the number of taps and the number of nonzero digits in each tap can be arbitrarily assigned given that enough hardware resource is available. The filter implemented with this architecture can be configured as a matched filter, a pulseshaping filter, or other filters. Furthermore, the proposed FIR architecture also has scalability, modularity, and cascadability, making it amenable to VLSI implementation.

The rest of the paper is organized as follows. In Section 2, the reconfigurable FIR filter architecture is presented. Circuit implementation of the reconfigurable FIR filter chip is then presented in Section 3. In Section 4, physical de- 
sign and measurement results of the chip are given and discussed. Finally, Section 5 concludes this paper.

\section{RECONFIGURABLE FIR FILTER ARCHITECTURE}

At first, note that an $N$-tap FIR filter can be described as

$$
y[n]=\sum_{i=0}^{N-1} h_{i} \cdot x[n-i] .
$$

If a coefficient, $h_{i}$, is expressed in the CSD format as $h_{i}=$ $\sum_{k=0}^{M_{i}-1} d_{i, k} \cdot 2^{-p_{k}}$, we can rewrite (1) as

$$
y[n]=\sum_{i=0}^{N-1} \sum_{k=0}^{M_{i}-1} d_{i, k} \cdot 2^{-p_{k}} \cdot x[n-i],
$$

where $d_{i, k} \in\{-1,0,1\}, p_{k} \in\{0, \ldots, L\} ; L+1$ is the length of the coefficients; and $M_{i}$ is the number of nonzero digits in $h_{i}$.

\subsection{Digit Processing Unit}

Most FIR filters that have been proposed are implemented using a tap as the basic building block. A tap is designed to evaluate the term $h_{i} \cdot x[n-i]$ in (1) and then several taps constitute an FIR filter. After examining (2) carefully, one sees that if a basic building block evaluates the term $d_{i, k} \cdot 2^{-p_{k}} \cdot x[n-i]$, then the flexibility on the number of taps and the number of nonzero digits in each tap can be achieved.

To meet this requirement, a digit processing unit (DPU), as shown in Figure 1, is designed. Control signals are serially shifted into a serial-in-parallel-out (SIPO) register (REG) array on the top during the initialization. In each DPU, three control signals, plus, zero, and shift, are derived from the corresponding digit in the $i^{\text {th }}$ tap coefficient, $d_{i, k} \cdot 2^{-p_{k}}$. The partial term, $d_{i, k} \cdot 2^{-p_{k}} \cdot x[n-i]$, is evaluated by the multiplier and the shifter. Another control signal, config, controls the multiplexer to select either the buffered or the unbuffered input as the output.

By cascading the DPUs, appropriately configuring the multiplexer in each DPU, and summing up the outputs of the DPUs and the accumulated sum as shown in Figure 2, we can implement an FIR filter with variable number of CSDs in each tap. For the last digit of each tap, the multiplexer in the corresponding DPU selects the buffered input as the output. Even though the architecture depicted in Figure 2 can be implemented directly, usually, it is necessary to insert pipeline registers in the filter to achieve reasonable speed performance.

\subsection{Reconfigurable FIR Filter Chip}

To illustrate the feasibility of the proposed reconfigurable FIR filter architecture, we designed a reconfigurable 8-DPU

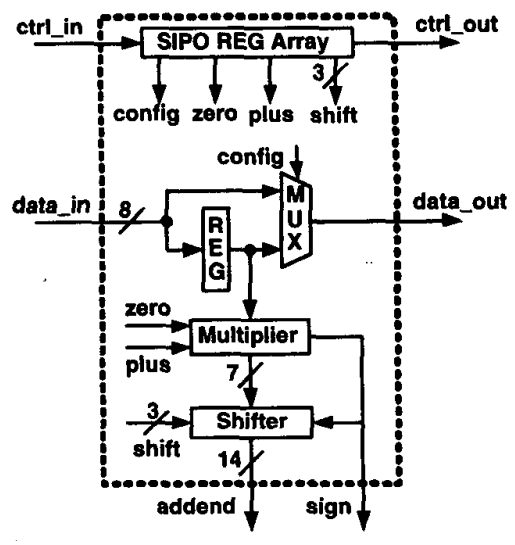

Figure 1: Digit processing unit.

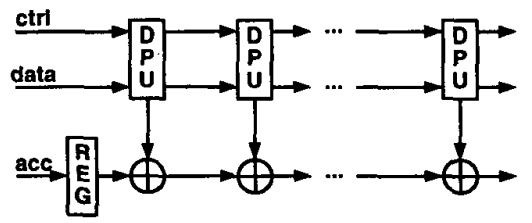

Figure 2: General reconfigurable FIR filter architecture.

FlR chip based on Figure 2. Detailed architecture is shown in Figure 3 and is referred to as a processing element (PE). In order to reduce the area and the latency required for implementing the addition in the filtering process, eight adders are combined to form a single big adder with nine inputs. With different precision in the output of the DPU (15-bit) and the accumulated sum (24-bit), we introduce a special sign extension generator. It generates the sum of sign extension bits of 8 DPUs' outputs based on the sign outputs of all DPUs. Finally, the addend outputs of 8 DPUs, the output of the sign extension generator, and the accumulated sum latched in the REG are summed together by an adder. One PE is implemented in the chip so that it is capable of at most 8-digit FIR computation. It is also designed to be cascadable so that the FIR filter with more taps can be implemented.

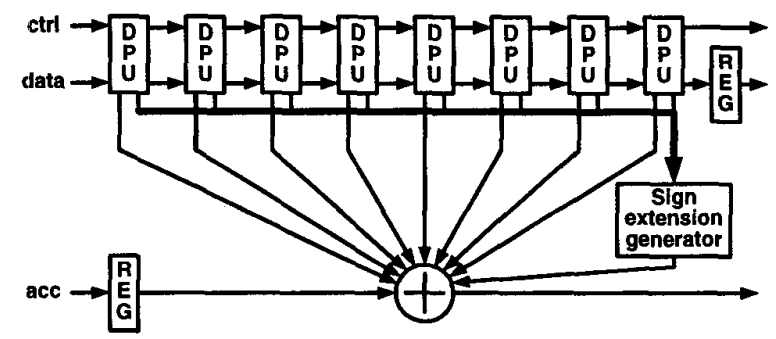

Figure 3: PE architecture. 
The reconfigurable FIR chip consists of one PE, one pseudo-random data generator (PRDG), and one test module (see Figure 4). There are two clock signals: CLK and DumpCLK, where CLK controls the operating speed of the filter and DumpCLK is used to initialize the chip parameters and to output the results. The parameters in each DPU are pre-calculated based on the filter configuration and then serially shifted into the chip with the ctrl_ in signal. Since the chip will be I/O limited, scan-chain is used for both testing and for normal partial sum communication between cascaded chips. The REG in the PE used to store the accumulated sum is replaced by a 24 -bit SIPO register array. The scan in signal is used to store the compensation vector explained below for the first chip or the accumulated sum of the previous chip into the 24-bit SIPO serially while cascading. A pseudo-random data generator is designed to provide the input test patterns in high-speed operation. The data fed into the first DPU can be generated by the pseudorandom data generator or data_in signal. Finally, due to the consideration of the output driving capability, a test module accumulates the 24-bit output of the adder in the PE with a 32-bit carry-save adder. The result can be serially scanned out at a slower clock rate to verify the functionality of the chip.

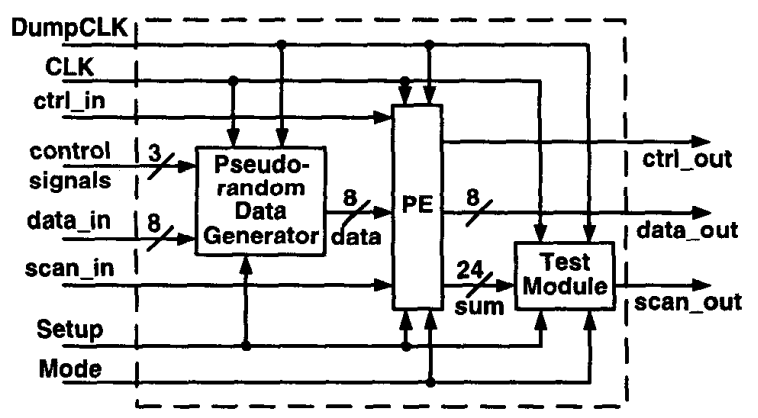

Figure 4: Chip block diagram.

\section{CIRCUIT IMPLEMENTATION}

\subsection{Multiplier and shifter}

The multiplier is used to multiply the input data $x[n-i]$ by $d_{i, k}$, which has three possible values: 1,0 , and -1 . The operation of each bit can be expressed as

$$
o u t p u t=\overline{z e r o+(p l u s \oplus i n p u t)}
$$

If $d_{i, k}$ is 0 , the zero signal will be ' 1 ' and force the output to be 0 regardless of the input. Otherwise, the zero signal will be ' 0 ' and (3) can be rewritten as output $=\overline{\text { plus } \oplus \text { input }}$. If the CSD coefficient is 1 , the plus signal will be ' 1 ' and the output is the same as the input. If the CSD coefficient is -1 , the plus signal will be ' 0 ' and the output is equivalent to the 1 's complement of the input. The one in LSB that needs to be added to form the 2's complement negation is accumulated for all negative digits. This sum forms the compensation vector and will be added by setting the initial value of the accumulator.

A shifter is used to multiply the term $d_{i, k} \cdot x[n-i]$ by $2^{-p_{k}}$ where $p_{k} \in\{0, \ldots, 7\}$. The shifter performs an arithmetic left shift and expands the 7-bit multiplier output (excluding the MSB) into a 14-bit output as the addend signal by shifting the input left by $7-p_{k}$ bits. Also, zeros are padded at LSB if the CSD coefficient is ' 1 ' or ' 0 ' and ones are padded if the CSD coefficient is ' -1 '.

\subsection{Sign extension generator}

In our architecture, the accumulated sum is 24-bit wide but the term $d_{i, k} \cdot x[n-i] \cdot 2^{-p_{k}}$ calculated by the DPU is only 15-bit wide (14-bit addend signal and 1-bit sign signal). While summing them together, it is unwise to extend each of them to the word length of the accumulated sum. It is better to deal with the sign extension bits separately, so that the area and power consumption can be reduced.

A sign extension generator is designed to evaluate the sum of sign extension bits based on eight sign signals. By examining the relation between the number of non-negative sign signals and the sum of the corresponding sign extension bits, a simple implementation of the sign extension generator is designed. The seven most significant bits can be selected through a MUX by examining if there exists any ' 1 ' valued sign signal. If the answer is yes, '1111111' will be selected, '0000000' will be selected otherwise. The three least significant bits are equivalent to the three least significant bits of the binary representation of the number of nonnegative sign signals.

\subsection{Adder}

The adder is used to sum eight 14-bit addend signals from DPUs, one 24-bit acc signal, and one 10-bit sign _ extend signal from the sign extension generator. The acc signal corresponds to the compensation vector or the accumulated sum stored in the REG shown in Figure 3. The acc signal is split into two parts where its fourteen LSBs and eight addend signals are compressed into four 14-bit signals by five 14-bit carry-save adders in a two-level arrangement. Its ten MSBs are then added with the sign_extend signal and the above four 14-bit signals by a two-level carry-save adder. Finally, an ELM adder [9] modified to reduce the critical path delay is used to compute the final sum.

\section{IMPLEMENTATIONS AND MEASUREMENTS}

Detail circuit design of the reconfigurable FIR filter chip was described in gate level using a hardware description lan- 
guage. Functional verification of the circuit design was conducted using five filters with different digit configurations. Layout was generated through a standard-cell-based design flow. Functional and timing simulations of the layout were carried out. The final layout is approximately $1.74 \times 1.64$ $\mathrm{mm}^{2}$ in a single-poly quadruple-metal $0.35-\mu \mathrm{m}$ CMOS technology and contains 27035 transistors. The die photo of the fabricated reconfigurable FIR filter chip is shown in Figure 5. There are 8 DPUs arranged in one row and three other blocks: an adder, a pseudo-random data generator, and a test module.

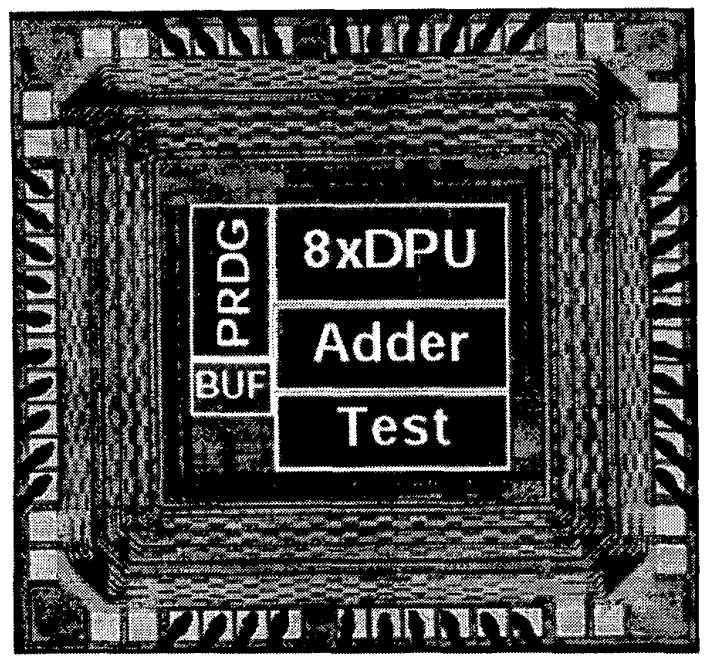

Figure 5: Chip die photo.

The fabricated chip has been tested and its function has been verified. The reconfigurable FIR filter chip can operate correctly at $86 \mathrm{MHz}$ under a supply voltage of $2.5 \mathrm{~V}$ and it consumes $16.5 \mathrm{~mW}$. The frequency of the DumpCLK is set to one-fourth of the operating frequency. Table 1 summarizes the major features of the reconfigurable FIR filter chip.

Table 1: Summary of the reconfigurable FIR filter chip.

\begin{tabular}{|c|c|}
\hline Clock Frequency & $86 \mathrm{MHz}$ \\
\hline Power Supply & $2.5 \mathrm{~V}$ \\
\hline Power Dissipation & $16.5 \mathrm{~mW}$ \\
\hline Process Technology & $0.35-\mu \mathrm{m} 1 \mathrm{P} 4 \mathrm{M} \mathrm{CMOS}$ \\
\hline Transistor Count & $27 \mathrm{~K}$ transistors \\
\hline Die Size & $1.74 \times 1.64 \mathrm{~mm}^{2}$ \\
\hline Package & 40 -pin S/B \\
\hline
\end{tabular}

\section{SUMMARY}

In this paper, a digit-reconfigurable FIR filter architecture is proposed. The design concepts of the architecture and the circuit are presented. Testing results show that the fabricated chip draws only $16.5 \mathrm{~mW}$ from a 2.5 -V power supply while running at $86 \mathrm{MHz}$.

\section{ACKNOWLEDGEMENT}

The authors greatly appreciate the Chip Implementation Center (CIC) of the National Science Council, Taiwan, R.O.C., for the fabrication and measurement of the proposed chip.

\section{REFERENCES}

[1] J. Mitola, "The Software Radio Architecture," IEEE Communications Magazine, vol. 33, pp. 26-38, May 1995.

[2] E. Buracchini, "The Software Radio Concept," IEEE Communications Magazine, vol. 38, pp. 138-143, Sept. 2000.

[3] R. M. Hewlitt and E. S. Swartzlantler Jr., "Canonical Signed Digit Representation for FIR Digital Filters," in Proc. of IEEE Workshop on Signal Processing Systems, 2000, pp.416-426.

[4] M. Tamada and A. Nishihara, "High-Speed FIR Digital Filter with CSD Coefficients Implemented on FPGA," in Proc. of the ASP-DAC, 2001, pp. 7-8.

[5] Y. M. Hasan, L. J. Karem, M. Falkinburg, A. Helwig, and M. Ronning, "Canonic Signed Digit Chebyshev FIR Filter Design,” IEEE Signal Processing Letters, vol. 8, pp. 167-169, June 2001.

[6] T. Zhangwen, Z. Zhanpeng, Z. Jie, and M. Hao, "A High-Speed, Programmable, CSD Coefficient FIR Filter," in Proc. of 4th International Conference on ASIC, 2001, pp. 397-400.

[7] K. T. Hong, S. D. Yi, and K. M. Chung, "A HighSpeed Programmable FIR Digital Filter Using Switching Arrays," in Proc. of IEEE Asia Pacific Conference on Circuits and Systems, 1996, pp. 492-495.

[8] K. Y. Khoo, A. Kwentus, and A. N. Willson Jr., "A Programmable FIR Digital Filter Using CSD Coefficients," IEEE Journal of Solid-State Circuits, vol. 31, pp. 869-874, June 1996.

[9] T. P. Kelliher, R. M. Owens, M. J. Irwin, and T. T. Hwang, "ELM-A Fast Addition Algorithm Discovered by a Program," IEEE Transactions on Computers, vol. 41, pp. 1181-1184, Sept. 1992. 